\title{
TEMPERATURE DEPENDENT MAGNETIZATION OF THE TWO BAND MODEL FOR DILUTED MAGNETIC SEMICONDUCTORS
}

\author{
VU KIM THAI \\ University of Economic and Technical Industries \\ HOANG ANH TUAN \\ Institute of Physics, Vietnam Academy of Science and Technology \\ E-mail: hatuan@iop.vast.ac.vn \\ Received 17 March 2014 \\ Accepted for publication 22 May 2014
}

\begin{abstract}
The temperature dependent magnetization of a two band model for diluted magnetic semiconductors as a function of magnetic coupling constant, hopping parameters and carrier densities is calculated by using the coherent potential approximation. It is shown that the degree of overlapping of the impurity bands and carrier density are crucial parameters determining the magnetization behavior of the system.
\end{abstract}

Keywords: magnetization; diluted magnetic semiconductors; two-band model; coherent potential approximation.

\section{INTRODUCTION}

The class of III-V diluted magnetic semiconductors (DMS) has recently attracted considerable attention after the experimental observation of high Curie temperature. These compounds can play an important role in spintronic devices, and they represent a challenge to theory due to combined presence of correlations and disorder.

The impurity band model, where $p$-holes move around interacting with localized spins at Mn sites through the antiferromagnetic exchange interaction, is a widely used model for (III,Mn) Vtype DMS [1-5]. In the one band model it has been found that the Curie temperature $T_{c}$ is maximized for the carrier density roughly half of the concentration of localized spins. In addition, for over doping of the carriers, $T_{c}$ decreases to zero. Recently the critical temperature was calculated using a two band model for DMS by Monter Carlo (MC) technique, dynamical mean field theory (DMFT) and coherent potential approximation (CPA) [6-7]. The main finding is that the maximal $T_{c}$ of the two band model is found when both impurity bands (IB) fully overlap and this value is approximately twice larger than the highest $T_{c}$ obtained in the single band model. Since the temperature dependence $M(T)$ of spontaneous magnetization possesses many characteristics, such as concavity/convexity of the curve, value of saturation magnetization, etc., its study has a very high potential for elucidating the physics behind DMS ferromagnetism in real systems. The purpose of this paper is to calculated the magnetization of localized spins as a function of temperature in the 
two band model. We show the degree of overlapping of the IB and carrier density to be the crucial parameters determining the magnetization behavior.

\section{THE MODEL AND FORMALISM}

We use a model of $\left(\mathrm{A}_{1-x} \mathrm{Mn}_{x}\right) \mathrm{B}$-type DMS where carriers hop among cation orbitals. We assume two orbitals at each cation site. The Hamiltonian reads

$$
H=\sum_{l i j \sigma} t_{l}\left(c_{l i \sigma}^{+} c_{l j \sigma}+\text { H.c. }\right)+\sum_{l, I} J_{l} \mathbf{S}_{I} \mathbf{s}_{l I},
$$

where $l=1,2$ is the band index, $t_{l}$ is the hopping term in the band $l, c_{l i \sigma}^{+}\left(c_{l j \sigma}\right)$ is the creation (annihilation) operator for a carrier at site $i$ with spin $\sigma$ in the band $l, \mathbf{s}_{l i}=c_{l i \alpha}^{+}\left(\frac{1}{2} \sigma_{\alpha \beta}\right) c_{l i \beta}$ is the spin operator at site $i$ of itinerant electron gas, $J_{l}$ is the coupling between the core spin and electrons of band $l$, and $\mathbf{S}_{I}$ is the spin of localized $\mathrm{Mn}$ ion at randomly selected sites $I$. In the present work we treat the localized spins as Ising spins $(S=1 / 2)$, though the extension to the case $S=5 / 2$ is straightforward [8-9].

The extension of CPA to the above model was presented in Ref. [7]. In this paper we apply this formulation to study the temperature dependent magnetization. In CPA the carriers are described as independent particles moving in an effective medium of spin-dependent coherent potentials. The coherent potential $\Sigma_{\sigma}(\sigma=\uparrow, \downarrow)$ is determined by demanding the scattering matrix for a carrier at an arbitrarily chosen site embedded in the effective medium vanished on average.

$$
(1-x)\left\langle t_{\sigma \sigma}^{A}\right\rangle_{T}+x\left\langle t_{\sigma \sigma}^{M n}\right\rangle_{T}=0 .
$$

Here the $t$-matrix elements $t_{\sigma \sigma}^{X}(X=A, M n)$ in the Wannier representation describe the multiple scattering of a $\sigma$-spin carrier by an $X$ ion. The thermal average at temperature for spin operators for any function $f\left(S^{z}\right)$ is given by

$$
\left\langle f\left(S^{z}\right)\right\rangle_{T}=\frac{\sum_{S^{z}=-S}^{S} f\left(S^{z}\right) \exp \left(\frac{h S^{z}}{k_{B} T}\right)}{\sum_{S^{z}=-S}^{S} \exp \left(\frac{h S^{z}}{k_{B} T}\right)},
$$

where $h$ denotes the effective field felt by the localized spins. By using a bare semicircular nonin-

teracting density of states (DOS) with halfbandwidth $W_{l}$ for the band $l: \rho_{0 l}(z)=\frac{2}{\pi W_{l}^{2}} \sqrt{W_{l}^{2}-z^{2}}$ from Eq. (2) we calculate the Green function and then DOS with spin $\sigma, \rho_{l \sigma}$, for each band for a given magnetization $\left\langle S^{z}\right\rangle_{T}=M$. The total DOS per site is given by $\rho_{t o t}(\omega)=\sum_{l \sigma} \rho_{l \sigma}(\omega)$, where $\rho_{l \sigma}=-1 / \pi \Im G_{l \sigma}(\omega)$. Throughout this work, we assume that the carriers are degenerate. Then the carrier density and the total energy can be expressed as

$$
n=\int_{-\infty}^{\mu} \rho_{t o t}(\omega) d \omega
$$




$$
E(M)=\int_{-\infty}^{\mu} \omega \rho_{t o t}(\omega) d \omega,
$$

where $\mu$ is the chemical potential. The free energy per site of the system is given as

$$
F(M)=E(M)-T S,
$$

where the entropy due to the localized spins is given by

$$
\mathcal{S}=x k_{B} \ln \sum_{S^{z}=-S}^{S} \exp \left(\frac{h S^{z}}{k_{B} T}\right)-\frac{h M}{T} .
$$

By minimizing $F$ with respect to $M$ we obtain the following equation for the effective magnetic field $h$

$$
h=-\frac{1}{x} \frac{d E(M)}{d M} .
$$

The local magnetization is then calculated by

$$
M=S B_{S}\left(\frac{h S}{k_{B} T}\right)
$$

where

$$
B_{S}(x)=\frac{2 S+1}{2 S} \operatorname{coth} \frac{2 S+1}{2 S} x-\frac{1}{2 S} \operatorname{coth} \frac{1}{2 S} x
$$

is the conventional Brillouin function. So we have a set of self-consistent equations for $\mu$ and $M$ for a given set of parameter values $x, n, J_{l}, W_{l}$ and $T$.

\section{NUMERICAL RESULTS AND DISCUSSION}

In this paper we take $W_{1}$ as the unit of energy. We have calculated the temperature dependent magnetization of localized spins $M(T)$ focusing on the doping $x=0.05$ associated with the highest $T_{c}$ in $\mathrm{Ga}_{1-x} \mathrm{Mn}_{x}$ As and varying exchange coupling constants, bandwidths and carrier densities. In Figs. 1 and 2 we show $M(T)$ for $W_{1}=W_{2}=J_{1}$ and four values of $J_{2}$ at $n=0.025$ (Fig. 1) and $n=0.05$ (Fig. 2). It can be seen in both figures that the Curie temperatures $T_{c}$ and the magnetization curves strongly depend on ratio $J_{2} / J_{1}$, i.e. the degree of overlapping of the IB. The magnetization curves are convex, but some look different from the classic textbook Weiss form. The $T_{c}$ is maximal for both $n$ when the bands fully overlap $\left(J_{2} / J_{1}=1\right)$ which agrees with [6,7]. In Figs. 3 and 4 we present $M$ versus $T$ at fixed $J_{1}=J_{2}=W_{1}$ varying bandwidths $W_{2} / W_{1}$ at $n=0.025$ (Fig. 3) and $n=0.05$ (Fig. 4). Again, the curves strongly depend on the degree of overlapping of the IB and $M(T)$ is maximal for all $T$ when the bands fully overlap $\left(W_{2} / W_{1}=1\right)$. Fig. 5 shows $M(T)$ when the IB fully overlap $\left(J_{1}=J_{2}, W_{1}=W_{2}\right)$ varying the carrier density. For different $n$ we have the linear behavior of $M(T)$ at low temperatures which qualitatively agree with the magnetization measurement in metallic $\mathrm{Ga}_{1-x} \mathrm{Mn}_{x} \mathrm{As}$ as mentioned in [10]. Additionally, one can see that in the two band model for all temperatures $M(T)$ is maximized at the carrier density roughly the concentration of localized spins $(n \approx x)$ which differs from the single band model where $M(T)$ is maximal for filling $n \approx x / 2$. As was noted in Ref. [6], the explanation is straightforward: at $n=x$ in the one band model the IB is fully occupied 
leading to a vanishing $T_{c}$, but for the same $n$ in the two band model both bands are half filled, which ultimately leads to the highest value for $T_{c}$ and $M(T)$.

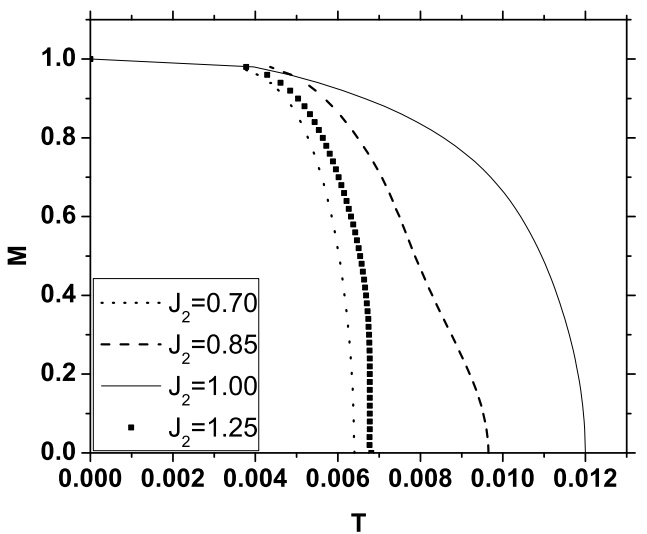

Fig. 1. Temperature dependent magnetization for various $J_{2} / J_{1}$ for $W_{1}=W_{2}=J_{1}=1$ and $n=0.025, x=0.05$.

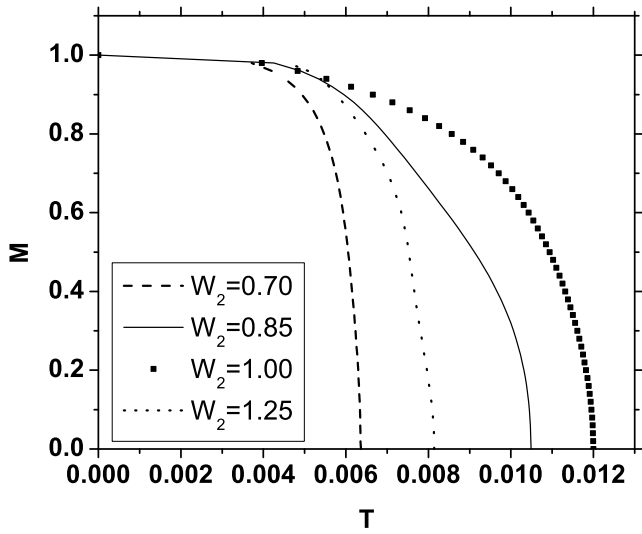

Fig. 3. Temperature dependent magnetization for various $W_{2} / W_{1}$ for $J_{1}=J_{2}=W_{1}=1$ and $n=0.025, x=0.05$.

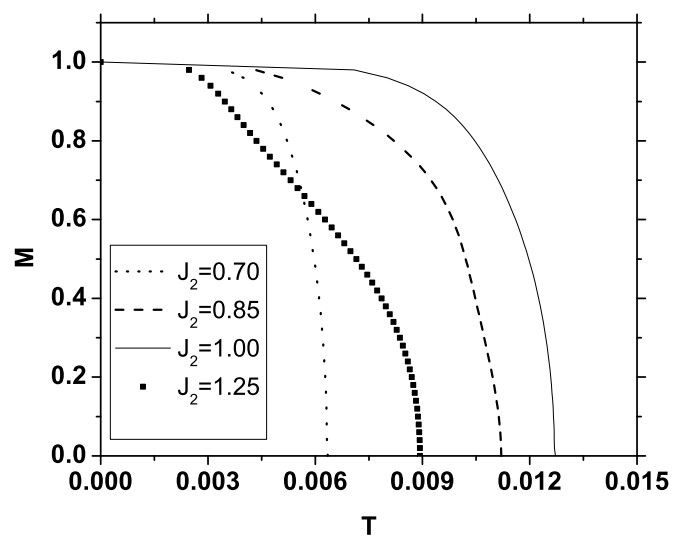

Fig. 2. Temperature dependent magnetization for various $J_{2} / J_{1}$ for $W_{1}=W_{2}=J_{1}=1$ and $n=x=0.05$.

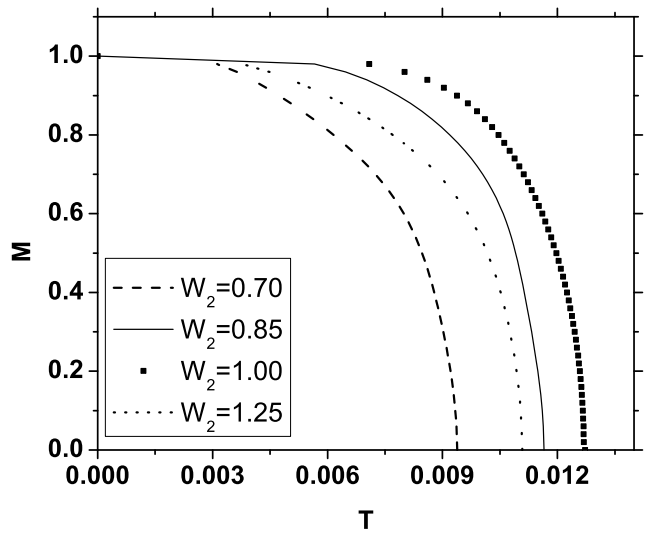

Fig. 4. Temperature dependent magnetization for various $W_{2} / W_{1}$ for $J_{1}=J_{2}=W_{1}=1$ and $n=x=0.05$.

In summary, in this paper the coherent potential approximation has been applied to the twoband model for DMS. The magnetization has been calculated by minimizing the free energy of the coupled carrier-localized spin systems. It has been shown the magnetization curves to be convex, the degree of overlapping of the IB and the carrier density to be crucial parameters determining the magnetization behavior. In particularly, we have found that when IB fully overlap $M(T)$ is maximized at $n=x$ and the magnetization curve is almost linear for low temperatures which are in good agreement with the ones obtained by DMFT [10]. In this work, we focus only on 
multi-band effects of DMS. There are, however, many features which exist in the real DMS but are not taken into account. Those are the band anisotropy, the nonmagnetic potential, the direct antiferromagnetic superexchange interaction between $\mathrm{Mn}$ ions and so on. These issues remain for future study.

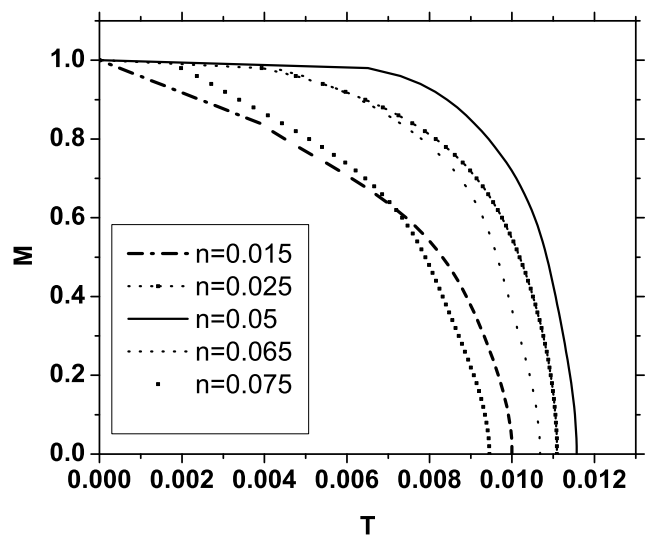

Fig. 5. Temperature dependent magnetization for various $n$ for $J_{1}=J_{2}=0.85, W_{1}=$ $W_{2}=1$ and $x=0.05$.

\section{ACKNOWLEDGMENTS}

This research is funded by Vietnam National Foundation for Science and Technology Development (NAFOSTED) under Grant No. 103.02-2011.05.

\section{REFERENCES}

[1] A.Chattopadhyay, S. Das Sarma and A. J. Millis Phys. Rev. Lett. 84 (2001) 227202.

[2] M. Yagi and Y. Kayanuma, J. Phys. Soc. Jpn. 71 (2002) 2010.

[3] G. Bouzerar, J. Kudrnovsky and P. Bruno, Phys. Rev. B68 (2003) 205331.

[4] M. Takahashi and K. Kubo, J. Phys. Soc. Jpn. 72 (2003) 2866.

[5] S. S. Feng and M. Mochena, Physica B 390 (2007) 79.

[6] F. Popescu, Y. Yildirim, G. Alvarez, A. Moreno and E. Dagotto, Phys. Rev. B73 (2006) 075206.

[7] Anh-Tuan Hoang, Mod. Phys. Letts. B 23 (2009) 3171.

[8] S. S. Feng and M. Mochena, J. Phys.: Condens. Matter 18 (2006) 1441.

[9] Anh-Tuan Hoang, Physica B 403(2008) 1803.

[10] S. Das Sarma, E. H. Hwang and A. Kaminski, Phys. Rev. B 67 (2003) 155201. 